\title{
Power consumption of piezoelectric actuators driving a simply supported beam considering fluid coupling
}

\author{
Steve C. Stein \\ AT\&T Bell Laboratory, 3000 Skyline Drive, Mesquite, Texas 75239 \\ Chen Liang \\ Department of Mechanical Engineering, San Diego State University, San Diego, California 92182-0101 \\ Craig A. Rogers \\ Center for Intelligent Material Systems and Structures, Virginia Polytechnic Institute and State University, \\ Blacksburg, Virginia 24061-0261
}

(Received 5 March 1993; revised 30 November 1993; accepted 13 May 1994) An electromechanical impedance model is applied to the case of a simply supported beam in an infinite rigid baffle with a fluid medium on one side. The effects of the fluid medium are included in the impedance analysis by considering fluid-structure interaction. The use of static and impedance model for structural acoustic analysis is discussed. Various power consumptions of PZT actuator-driven underwater beam structures will be quantified. The analysis discussed in this paper will be used to determine radiated structural acoustic power without using microphones. This work is the first step toward the determination of power requirements for underwater active structural acoustic control.

PACS numbers: $43.40 . \mathrm{Vn}, 43.38 . \mathrm{Fx}$

\section{INTRODUCTION}

Active structural acoustic control (ASAC) using induced strain actuators has recently gained attention as a possible means of controlling low-frequency noise $(<1000 \mathrm{~Hz})$. ASAC has been demonstrated experimentally for composite beams with embedded shape memory alloy (SMA) actuators (Rogers, 1990; Saunders et al., 1990), beams with bonded piezoelectric (PZT) actuators (Fuller et al., 1990) and plates with bonded PZT actuators (Clark and Fuller, 1992). Other research has focused on optimization of multiple PZT actuators for ASAC of plates (Wang, 1991) and ASAC of cylindrical shells using PZT actuators (Lester and Lefebvre, 1991; Sonti et al., 1991). These efforts have been useful in identifying the significant design parameters for an induced strain actuator utilized in an ASAC system (i.e., size, location, thickness, phasing, etc.).

In most, if not all, of the research related to ASAC with induced strain actuators, the investigation of energy consumption has been ignored. For thin beam, plate, and shell structures the energy consumption may not be a concern; however, for larger, complicated structures, such as marine vessels, which may require a great number of relatively large actuators, the power consumed by the actuators may be very significant. High-actuator power consumption signifies large power supplies which are expensive and massive. Reducing the power consumption of the systems, therefore, will reduce both the cost and mass.

In ASAC with PZT actuators, the sound pressure is linearly related to the applied forces and moments. Increasing the sound pressure level in $\mathrm{dB}$ is therefore limited by the amount of induced force/moment the actuator can deliver. The voltage level and power consumption, however, can be significantly reduced by increasing the force/moment output of the actuator for a set electric field. One way of increasing the moment of a bonded actuator without changing the applied electric field is to offset the actuator from the structure (Chaudhry and Rogers, 1992). As an example, consider an actuator configured in such a way as to obtain a $100 \%$ increase in induced moment. This increase in moment increases the sound pressure level by only about $3 \mathrm{~dB}$. However, the voltage level needed to maintain the original sound pressure level will drop by $50 \%$ and the power consumption can be reduced by up to $75 \%$. Thus, power consumption of actuators should be included as a primary variable in the objective function for optimization of intelligent material systems for active control. This paper uses a generic electromechanical impedance model (Liang et al., 1993a, 1993b), for determining the power consumed by PZT actuators for underwater ASAC.

\section{INTRODUCTION TO THE ELECTROMECHANICAL IMPEDANCE MODEL}

The electromechanical impedance model considers any electrically driven active structure an electrical and mechanical network system coupled by two-port electromechanical energy conversion devices, i.e., actuators. The electromechanical system behavior may be characterized by its electromechanical admittance and the dynamic interaction forces. The interactions between actuators and structures must be determined based on the dynamic output characteristics of the actuators and the dynamic characteristics of the structure, i.e., the structural impedance. Mass, damping, rigidity, boundary conditions, and acoustic medium all contribute to the impedance of a structure. The model may be used to determine the dynamic response and power consumption of actuators used in active structures.

Extensive experiments have been conducted to verify the electromechanical impedance model. These experiments 


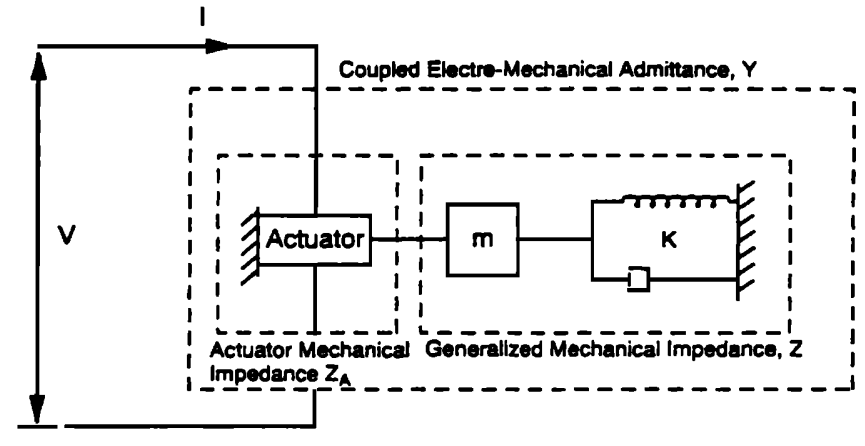

FIG. 1. An electromechanical representation of PZT actuator-driven mechanical systems. The mass, spring, damper, and boundary conditions shown in the figure indicate the structural components that contribute to the structural impedance.

include dynamic response, power consumption, and transmitting force measurement (Rossi et al., 1993; Zhou et al., 1994a, 1994b). The tested host structures include beams, rings, plates, and cylinders. The extension of the electromechanical impedance model to two-dimensional structures, such as cylinders and plates, may be found in Zhou et al. (1994a,b).

Consider a PZT actuator-driven one-degree-of-freedom spring-mass-damper system, as shown in Fig. 1. The electrical field is applied in the $z$ direction (3-3 direction), and the actuator expands and contracts only in the $y$ direction (2-2 direction). The force transmitted from the actuator varies as a function of frequency and is related to the mechanical impedances of the actuator and the structure by

$$
\bar{F}=w_{A} h_{A} \bar{T}_{2_{y=l_{A}}}=-\frac{Z}{\left(Z_{A}+Z\right)} d_{32} \bar{E} \bar{Y}_{22}^{E} w_{A} h_{A},
$$

where $\bar{T}_{2}$ is the stress in the actuator, $w_{A}$ is the width, $h_{A}$ is the thickness, $d_{32}$ is the piezoelectric coefficient, $\bar{Y}_{22}^{E}=Y_{22}^{E}(1+i \delta)$ is the complex elastic modulus at a constant electrical field, $\delta$ is the mechanical loss factor for the $\mathrm{PZT}, \bar{E}$ is the applied electric field, and $Z$ is the mechanical impedance of the structure. The short-circuit actuator mechanical impedance $Z_{A}$ is

$$
Z_{A}=\frac{K_{A}}{i \omega} \frac{k l_{A}}{\tan \left(k l_{A}\right)},
$$

where $K_{A}$ is the stiffness of the PZT actuator given by $\bar{Y}_{22}^{E} w_{A} h_{A} / l_{A}$ and $k^{2}=\omega^{2} \rho / \bar{Y}_{22}^{E}$. The structural impedance varies depending on the type of structure. The transmitting force of the PZT is used to determine the dynamic response of the integrated actuator/structure system which will be discussed later in this section.

From the constitutive relation of piezoelectricity (charge as a function of applied stress and electrical field), the electrical displacement of a PZT actuator may be determined, which is frequency dependent. The electrical current passing through the PZT actuator can be determined by integrating the charge over the actuator surface. The coupled electromechanical admittance, defined as current over voltage, pro- vides the link between the actuator and structure impedances and the electrical aspects of the system. The electromechanical admittance (defined as $Y=I / V$ ) is

$$
Y=i \omega \frac{w_{A} l_{A}}{h_{A}}\left(\bar{\epsilon}_{33}^{T}-\frac{Z}{Z_{A}+Z} d_{32}^{2} \bar{Y}_{22}^{E}\right),
$$

where $\bar{\epsilon}_{33}^{T}$ is the complex dielectric constant of the PZT actuator in the 3-3 direction under a constant stress. The complex dielectric constant is given by $\epsilon_{33}^{T}(1-\eta i)$, where $\eta$ is the dielectric loss factor.

The influence of the structure and the actuator impedance is clear in the equations above. The actuator electrical power is related to the admittance through the following.

The apparent power, $W_{A}$ :

$$
W_{A}=\frac{V^{2}|Y|}{2},
$$

the dissipative power, $W_{D}$ :

$$
W_{D}=\frac{V^{2} \operatorname{Re}(Y)}{2},
$$

and the reactive power, $W_{R}$ :

$$
W_{R}=\frac{V^{2} \operatorname{Im}(Y)}{2} \text {. }
$$

The dissipative power is discussed in this paper. For a mechanical system vibrating in a vacuum, the power consumed includes three parts: the first two result from the mechanical and dielectric losses in the PZT itself, and the third results from the damping in the mechanical system. For a system with fluid loading, the additional impedance from the interaction of the structure with the fluid medium causes an increase in power consumption of the actuator.

Everything in Eqs. (1) and (3) is known except for the structural mechanical impedance $Z$. The other terms in the equations are functions of the electrical and mechanical properties of the PZT actuator. After determining the structural impedance, the dynamic response and power consumption of the actuator may be found. The expression for admittance in Eq. (3) is general and holds for any system; thus, the equations can be applied to a continuous system simply by replacing the impedance of the spring-mass-damper system with the driving point structural impedance of a continuous system. The driving point structural impedance may be determined using any theoretical approach or even from experiments. The structural impedance for an underwater simply supported beam excited by two PZT patch actuators bonded on its top and bottom, as shown in Fig. 2, is developed in the subsequent section.

\section{STRUCTURAL IMPEDANCE OF A BAFFLED UNDERWATER SIMPLY SUPPORTED BEAM}

This section develops the structural impedance for a continuous system, namely a baffled beam and its acoustic medium. The emphasis is on the effect of the fluid loading on the structural impedance of the actuator/beam system and, subsequently, the effect on the power consumption of the actuator. Consider the baffled simply supported beam as 


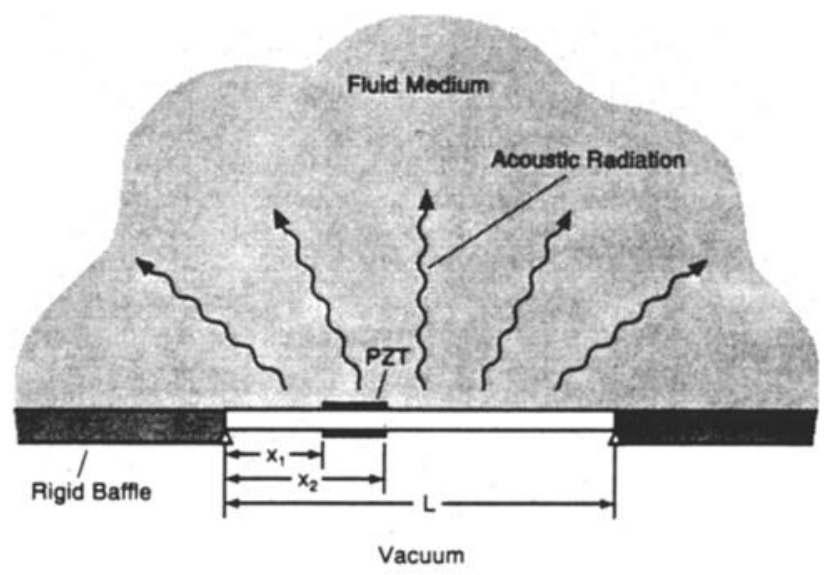

FIG. 2. A baffled underwater simply-supported beam with surface-bonded PZT actuators.

shown in Fig. 2. The beam is surrounded by an infinite rigid baffle and radiates sound into an acoustic fluid on one side. The beam has a modulus of elasticity $E$, mass density $\rho$, and moment of inertia over area $k_{i}^{2}$. Damping is included in the model through the use of a complex modulus, $E(1+i \delta)$, where $\delta$ is the mechanical loss factor.

The equation of motion for the transverse deflection $v(x, t)$ in the presence of an acoustic fluid is

$$
\rho \frac{\partial^{2} v}{\partial t^{2}}=-(1+i \delta) E k_{i}^{2} \frac{\partial^{4} v}{\partial x^{4}}+\frac{p(x)}{h}-\frac{p_{i}}{h},
$$

where $h$ is the thickness of the beam, $p(x)$ is the external dynamic loading, and $p_{i}$ is the acoustic pressure (unit: $\mathrm{N} / \mathrm{m}^{2}$ ).

The governing equation, Eq. (7), is solved by expanding the transverse displacement and external loads in terms of the eigenfunctions of the system without fluid loading. The applied forcing function is harmonic and can be expressed as

$$
p(x, t)=\sum_{m=1}^{\infty} P_{m} \chi_{m}(x) \exp (i \omega t),
$$

where $\omega$ is the driving frequency and

$$
\chi_{m}=\sin (m \pi x / L)
$$

is the eigenfunction of a simply supported beam of length $L$. The transverse displacement may be expressed as

$$
v=\sum_{m=1}^{\infty} W_{m} \chi_{m}(x) \exp (i \omega t) .
$$

A detailed derivation of the steady-state acoustic pressure from a flat, elastic plate is given by Sandman (1977), which is modified and used for this analysis. It is necessary to state that the acoustic pressure results presented by Sandman (1977) may not be directly applied to a simply supported beam because of the free boundary conditions in the $y$ direction. However, since the objective here is to demonstrate an analysis technique and its potential utility, the approximation here is considered to be acceptable. A more comprehensive and accurate analysis on this topic dealing with underwater plates conducted by the authors may be found in Liang et al.
(1994). The acoustic pressure on the beam approximated from Sandman's results may be expressed as

$$
\begin{aligned}
p_{i}(x, y, t)= & -\frac{\rho_{f} \omega^{2}}{2 \pi} \sum_{m=1}^{\infty} W_{m} \int_{0}^{b} \int_{0}^{L} \chi_{m}\left(x_{1}\right) \\
& \times \frac{\exp (-i k R)}{R} d x_{1} d y_{1} \exp (i \omega t),
\end{aligned}
$$

where $k$ is the acoustic wave number $\omega / c_{f}, c_{f}$ is the acoustic wave speed, and $\rho_{f}$ is the fluid density. In Eq. (11), $R$ is given by

$$
R=\sqrt{\left(x-x_{1}\right)^{2}+\left(y-y_{1}\right)^{2}} .
$$

The pressure given by Eq. (11) varies in the width direction of the beam. The following relation is utilized to calculate the average pressure in the width direction:

$$
p_{i}(x, t)=\frac{1}{w} \int_{0}^{w} p_{i}(x, y, t) d y .
$$

Substituting Eqs. (8), (10), and (13) into Eq. (7), multiplying by $\chi_{n}$, and integrating over the length of the beam gives

$$
A_{m} W_{m}+B \sum_{n=1}^{N} \gamma_{m n} W_{n}=\frac{1}{\rho h} P_{m},
$$

where

$$
\begin{aligned}
& A_{m}=c^{2} k_{i}^{2}(m \pi / L)^{4}-\omega^{2} \\
& B=-\rho_{f} \omega^{2} / \pi \rho h w L
\end{aligned}
$$

and

$$
\begin{aligned}
\gamma_{m n}= & \int_{0}^{b} \int_{0}^{L} \int_{0}^{b} \int_{0}^{L} \sin \left(\frac{n \pi x_{1}}{L}\right) \sin \left(\frac{m \pi x}{L}\right) \\
& \times \frac{\exp (-i k R)}{R} d x_{1} d y_{1} d x d y
\end{aligned}
$$

In Eq. (15), $c$ is the complex wave speed of the beam given by $[E(1+i \delta) / \rho]^{1 / 2}$.

For a pair of PZT patch actuators bonded on the top and bottom of a beam, the equivalent excitation may be modeled as a pair of moments at the ends of the PZT actuators for out-of-phase activation assuming the length of the PZT actuator is much larger than its thickness. The internal moment (within the beam) resulting from the equivalent moment loading may be expressed as

$$
M(x)=M_{T}\left[H\left(x-x_{2}\right)-H\left(x-x_{1}\right)\right],
$$

where $M_{T}$ is the transmitting moment (per unit width) from the activation of the PZT actuator, which is unknown at this time, $H(x)$ is the Heaviside function, and $x_{1}$ and $x_{2}$ are the coordinates of the two ends of the actuators.

The transmitting moment $M_{T}$ is assumed to be unity in the calculation of the driving point structural impedance. Once the structural impedance (consider with fluid interaction) is determined, the actual transmitting moment from the PZT actuator to the beam can be determined based on the 
impedance model described earlier. The unit equivalent actuator pressure loading, $p(x)$, resulting from the activation of the PZT actuator, may be expressed as

$$
p(x)=\frac{d^{2} M(x)}{d x^{2}} .
$$

The modal coefficient of the external loading, $P_{m}$, for the unit actuator loading can be determined as (Wang, 1991)

$$
P_{m}=\frac{2 m \pi}{L^{2}}\left[\cos \left(\frac{m \pi x_{2}}{L}\right)-\cos \left(\frac{n \pi x_{1}}{L}\right)\right] .
$$

Equation (14) may be written in a matrix form as

$$
([A]+B[\gamma])\{W\}=(1 / \rho h)\{P\},
$$

which describes a set of linear equations that can be solved for the modal amplitudes, $W_{m}$. For a beam in a vacuum, the fluid density $\rho_{f}$ is zero (thus the coefficient $B$ is zero). Since matrix $[A]$ is diagonal, the modal amplitudes $W_{m}$ can easily be solved as

$$
W_{m}=P_{m} / \rho A_{m} h .
$$

For a beam with fluid loading, $[\gamma]$ must be obtained using numerical integration and $W_{m}$ is found using

$$
\{W\}=(1 / \rho h)([A]+B[\gamma])^{-1}\{P\} .
$$

At this point, the displacement frequency response function corresponding to the unit actuator activation is determined by substituting the modal amplitudes, $W_{m}$, into Eq. (10). The acoustic power frequency response function corresponding to the unit excitation force $\bar{\Pi}$ is found by integrating the acoustic intensity over the semi-infinite hemisphere. For both the uncoupled and fluid-coupled analyses, the driving point structural impedance corresponding to the general loading of the PZT actuator (a pair of bending moments) may be expressed as (Liang et al., 1993a):

$$
Z=\frac{\left(h_{a}+h\right)^{2}}{2 i \omega\left[\theta\left(x_{2}\right)-\theta\left(x_{1}\right)\right]},
$$

where $\theta\left(x_{1}\right)$ and $\theta\left(x_{2}\right)$ are the rotation of the beam at $x_{1}$ and $x_{2}$, respectively. The generalized impedance given by Eq. (24) represents the dynamic resistance of the beam to the activation of the PZT actuator (a pair of moments versus curvature variation) and can be directly used in Eqs. (1) and (3).

Once the structural impedance is known, the transmitting force from the actuator, the electro-mechanical admittance of the actuator, and the power consumption of the actuator are computed from the expressions given in Eqs. (1) to (6).

One of the important ideas presented in this paper is that the radiated acoustic power may be determined by measuring the power consumption of the actuators that excite the structures. This concept is straightforward based on energy conservation principles but would be very difficult to experimentally verify. A simple theoretical verification may be conducted by comparing the radiated acoustic power obtained by integrating the far-field acoustic intensity and the one predicted based on the actuator power consumption cal- culation. The radiated acoustic power may be easily determined once $W_{m}$ is obtained from Eq. (23) using the techniques discussed in Roussos (1985).

Since the acoustic power is proportional to the square of the excitation force, the actual acoustic power can be found by multiplying the acoustic power resulting from a unit actuator excitation, $\bar{\Pi}$, by the square of the transmitted moment from the actuator, $M_{T}$, as

$$
\Pi=\bar{\Pi}\left|M_{T}\right|^{2} .
$$

Here, $\bar{\Pi}$ is also called the power frequency response function, which may be determined when calculating the structural impedance. The transmitting moment can be determined by multiplying the thickness of the beam by the transmitting force obtained from Eq. (1).

\section{IMPEDANCE AND STATIC APPROACH}

The static approach refers to the method of using a statically equivalent set of forces and moments as the amplitude of the forcing function in determining the dynamic response of actuation from integrated actuators. For example, the equivalent static moment from two PZT actuator patches bonded on a beam, such as the ones shown in Fig. 2, may be expressed as (Wang, 1991):

$$
M_{e q}=\frac{t_{b}^{2} E_{b}}{6+t_{b} E_{b} / t_{a} E_{a}} w \Lambda,
$$

where $t_{a}$ and $t_{b}$ are the thicknesses of the actuator and beam, respectively. Here, $E_{a}$ and $E_{b}$ are the modulus of the actuator and beam, respectively, $w$ is the width of the beam (or actuator), and $\Lambda$ is the free induced strain of the actuator. Equation (26) describes the interaction moment determined from an infinite large beam considering static force equilibrium and geometrical compatibility. Some dynamic analyses of structures driven by induced strain actuators use the moment given by Eq. (26) as the forcing function, while neglecting the stiffness and mass loading from the actuators. This approach (using a statically determined moment as the equivalent forcing function) has been used to determine structural response and acoustic radiation. The analysis presented in this paper will show that the use of the static approach may result in some errors in the dynamic analysis of structure with integrated induced strain actuators.

\section{NUMERICAL EXAMPLE}

Figure 2 shows a simply supported beam with two PZT patch actuators bonded on the top and bottom surfaces. The actuators are activated out-of-phase, imparting pure bending excitation. The beam is $38 \mathrm{~cm}$ long, $4 \mathrm{~cm}$ wide and $2 \mathrm{~mm}$ thick with a modulus of elasticity $63 \mathrm{GPa}$ and loss factor of $0.5 \%$. The beam is enclosed by an infinite rigid baffle and radiates acoustic energy into a fluid medium on one side. The left end of the PZT actuators is at $7.9 \mathrm{~cm}$. The actuators are $3.8 \mathrm{~cm}$ long, $4 \mathrm{~cm}$ wide, and $0.2 \mathrm{~mm}$ thick. The electromechanical properties of the PZT material are listed in Table I.

A FORTRAN code was written to perform the necessary numerical integrations and modal summations to compute the fluid-coupled structural impedance of the beam. Ten 
TABLE I. Electrical and mechanical properties of the PZT material (G1195).

\begin{tabular}{cccccc}
\hline \hline $\begin{array}{c}d_{32} \\
(\mathrm{~m} / \mathrm{V})\end{array}$ & $\begin{array}{c}Y_{22}^{E} \\
\left(\mathrm{~N} / \mathrm{m}^{2}\right)\end{array}$ & $\begin{array}{c}\rho \\
\left(\mathrm{kg} / \mathrm{m}^{3}\right)\end{array}$ & $\begin{array}{c}\epsilon_{33}^{T} \\
(\text { Farads/m) }\end{array}$ & $\eta$ & $\delta$ \\
\hline$-166 \times 10^{-12}$ & $6.3 \times 10^{10}$ & 7650 & $1.5 \times 10^{-8}$ & 0.012 & 0.05 \\
\hline
\end{tabular}

modes are used in the modal expansion to provide reasonable accuracy. The fluid densities are $1.21 \mathrm{~kg} / \mathrm{m}^{3}$ and $1000 \mathrm{~kg} / \mathrm{m}^{3}$ for air and water, respectively. The nominal sound speed in air is $343 \mathrm{~m} / \mathrm{s}$ and $1500 \mathrm{~m} / \mathrm{s}$ in water.

\section{RESULTS AND DISCUSSION}

The top chart of Fig. 3 shows that the transmitting moment from the actuators to their host beam, predicted using the impedance model (solid line), is frequency dependent. The dashed line is the static moment predicted using Eq. (26). The radiated sound power results calculated from the impedance model can be obtained from the static model results using

$$
\Pi=\Pi_{\text {static }} \frac{\left|M(\omega)_{\text {impedance }}\right|^{2}}{\left|M_{\text {static }}\right|^{2}} .
$$

Equation (27) is the same as Eq. (25). The radiated acoustic power predicted by the static model divided by the square of the static interaction moment yields the power frequency response function used in Eq. (25).

The bottom chart of Fig. 3 compares the radiated acoustic power calculated using both the impedance approach (solid line) and the static approach (dashed line). It is evident from the curves that the force behavior is reflected in the radiated acoustic power. The radiated sound power for both impedance and static model are determined by integrating the sound intensity over the semisphere in the fluid medium.

Figure 4 illustrates the essence of the dynamic interaction between the actuators and their host structures. The top
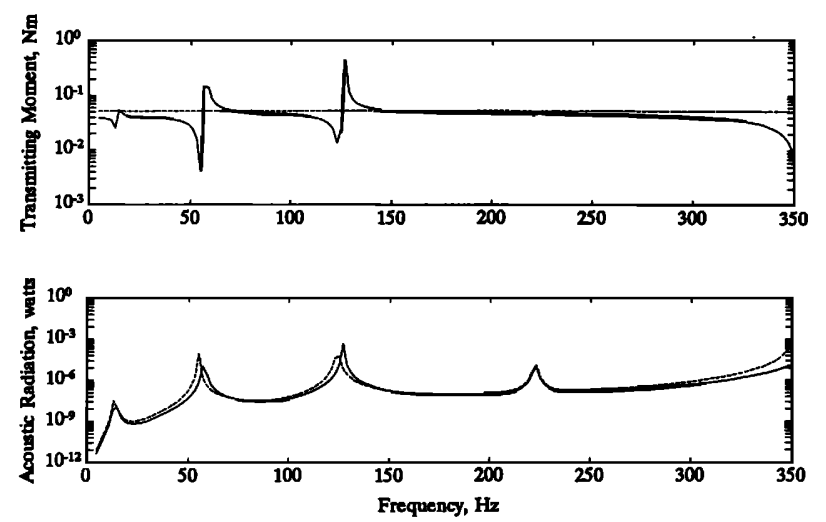

FIG. 3. Comparison of the impedance and static models. The top chart illustrates the dynamic interaction force (transmitting moment) determined based on the impedance model. The dashed line is the static interaction moment predicted using Eq. (26). The bottom chart shows the radiated acoustic power predicted using the impedance method (solid line) and static approach (dashed line). The radiated acoustic power is calculated by integrating the acoustic intensity over the semisphere in the fluid medium. The fluid coupling (in water) is included in this analysis. Similar results may also be obtained for in-air analysis.
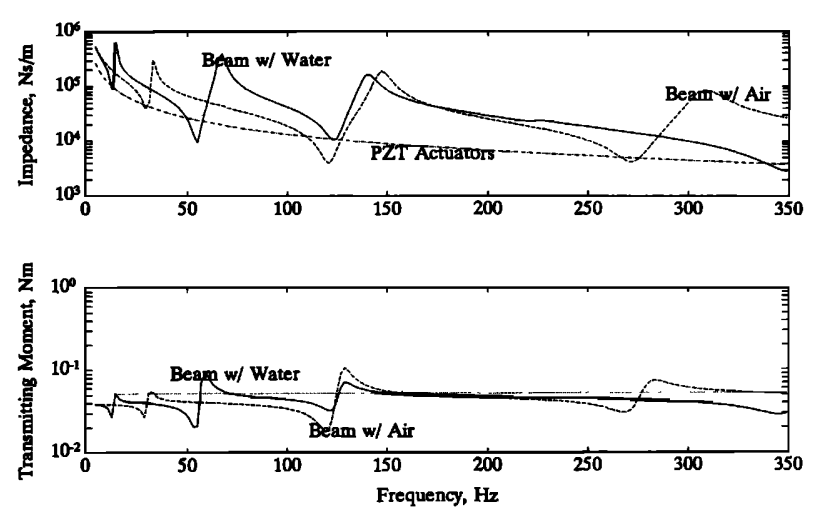

FIG. 4. Essence of the dynamic interaction between the actuators and their host beam. The top chart shows the driving point fluid-coupled mechanical impedance [Eq. (24)] for in-water (solid line) and in-air (dashed line) case studies. The short circuit actuator impedance is plotted as the dash-dotted line. The structural impedance and actuator impedance become a pair of complex conjugates (match) at the intersection point of the rising structural impedance curve and actuator impedance curve. The bottom chart depicts the resulting transmitting moment for in-water (solid line) and in-air (dashed line) case studies. The static moment predicted from Eq. (26) is also plotted (dotted line). The frequency dependency of the dynamic transmitting moment is a result of the structural impedance interaction as indicated by Eq. (1).

chart of Fig. 4 plots the fluid-coupled mechanical impedance at the driving point according to Eq. (24). The solid line is for the in-water case and the dashed line is for the in-air case. The short-circuit mechanical impedance of the actuator given by Eq. (2) is also plotted (dash-dotted line). The bottom chart of Fig. 4 shows the transmitting moment for in-water (solid line), in-air (dashed line), and the static moment from Eq. (26) (dotted line). In general, the dynamic interaction moment is close to the static moment except around the resonance of the beam and actuators where the beam and actuator impedance curves intersect (match in terms of complex conjugates). More detailed discussion on this topic may be found in other papers by the authors listed in the references.

Clearly, the impedance model provides more physical insight into the dynamic actuator-structure interaction than the static models. Use of the static model for predicting dynamic behavior is ill-advised as attested by the results of Figs. 3 and 4. While the results show a general correlation in shape, the natural frequencies differ (due to actuator stiffening and mass loading), as do the sound power amplitudes, which are off by about an order of magnitude at some frequencies. Static models, therefore, should be avoided when calculating dynamic and acoustic responses for active material systems, especially when the actuator impedance is close to the structural impedance.

The predictions of actuator power consumption and its breakdown for underwater acoustic radiation are shown in Figs. 5 and 6. The dashed line in Fig. 5 is the actuator power consumption due to acoustic radiation calculated from Eqs. (3) and (5), which is almost identical to the acoustic power calculated by integrating the acoustic intensity (the solid line in the bottom chart of Fig. 4). The solid line in Fig. 5 is the power dissipation due to acoustic radiation and the mechanical loss of the beam. The difference between the two curves 


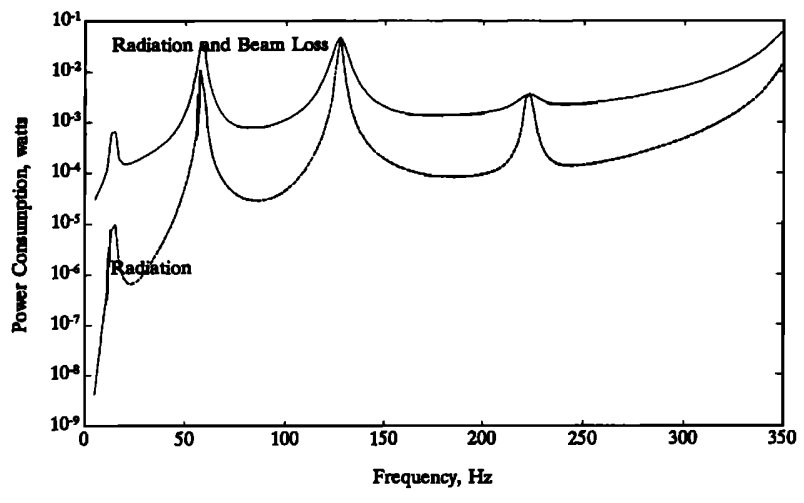

FIG. 5. Actuator power consumption due to acoustic radiation and structural damping. The solid line is the power dissipation resulting from acoustic radiation and the internal mechanical loss of the beam. The dashed line represents the power consumption because of structural acoustic radiation. The acoustic radiation result here is calculated using Eqs. (3) and (5), which is identical to the one obtained by integrating the acoustic intensity given by the solid line in the bottom chart of Fig. 3.

may be used to determine the power dissipation due to structural damping.

The solid line shown in Fig. 6 is the total actuator power consumption resulting from the dielectric loss (dashed line), the mechanical loss of the actuator (dash-dotted line), and the acoustic radiation and structural loss (solid line in Fig. 5). To determine the individual effect of a single loss effect, models were run with the other loss parameters set to zero. For example, to determine the power consumed by structural damping of the beam, the dielectric loss and mechanical loss factors of the PZT were set to zero, as was the density of the acoustic medium.

An interesting potential application exists for the impedance model along with the power consumption analysis. The total actuator power consumption may be easily measured. The loss due to actuator dissipation (electrical and mechanical loss) may be determined based on the characteristics of the actuator. The actuator dissipation can then be used to modify the measured total power consumption, yielding the

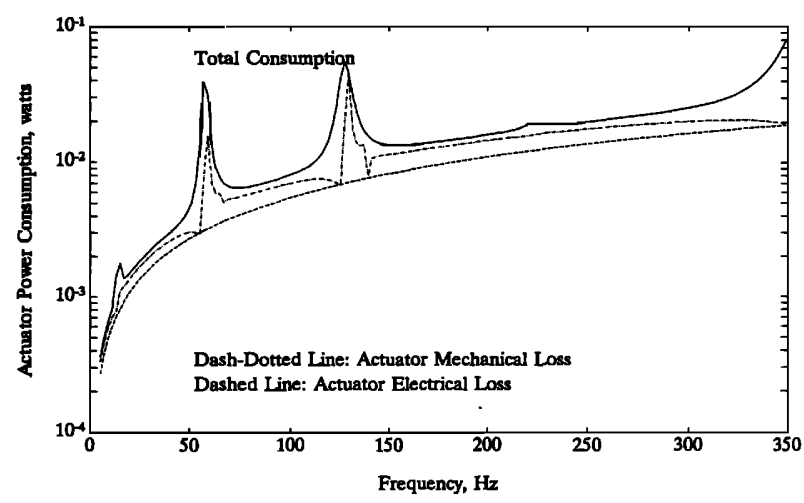

FIG. 6. Breakdown of actuator power consumption due to electrical loss (dielectric loss for PZT materials given by the dashed line) and mechanical loss (dash-dotted line). The solid line is the total actuator power consumption (a measurable quantity), which may also be determined by summing the power dissipation resulting from actuator electrical and mechanical loss, structural mechanical loss, and acoustic radiation. power dissipated due to structural damping and acoustic radiation (the solid line in Fig. 5). If the experiment is conducted in a vacuum, which eliminates the influence of acoustic radiation on power dissipation, the measured structural loss characteristics can be used to modify the solid line in Fig. 5 to calculate radiated acoustic power. This will be an acoustic measurement without using microphones and may be important in underwater structural acoustic control where using far-field microphones to provide error signals is not practical.

\section{CONCLUSIONS}

This paper extends the electromechanical impedance approach for analyzing the energy transfer and power consumption in active material systems to the case of acoustic radiation in the presence of a fluid. The approach uses a structural impedance method to find radiated acoustic power and power consumption. The numerical results demonstrate the significance of accounting for fluid coupling when determining the structural impedance of systems and the power consumption of actuators used in intelligent material systems. In addition, the numerical results show the inadequacy of static models for predicting dynamic response and power consumption. The capability to analyze the power consumption and energy transfer of actuators utilized in underwater acoustic control will enable the design of more energyefficient active control systems.

\section{ACKNOWLEDGMENTS}

The authors gratefully acknowledge the support of the Office of Naval Research, Grant No. ONR-00014-92-J-1170, Dr. Kam, Ng, Technical Monitor.

Chaudhry Z., and Rogers, C. A. (1992). "Enhanced Structural Control with Discretely Attached Induced Strain Actuators," Proceedings of the 33rd SDM Conference, Dallas, TX (1992).

Clark, R. L., and Fuller, C. R. (1992). "Experiments on Active Control of Structurally Radiated Sound Using Multiple Piezoceramic Actuators," J. Acoust. Soc. Am. 91, 3313-3320.

Fuller, C. R., Gibbs, G. B., and Silcox, R. J. (1990). "Simultaneous Active Control of Flexural and Extensional Waves in Beams," J. Intell. Mater. Syst. Struct. 1(2), 235-247.

Lester, H. C., and Lefebvre, S. (1991). "Piezoelectric Actuator Models for Active Sound and Vibration Control of Cylinders," Proceedings: Recent Advances in Active Control of Sound and Vibration (Technomic, Lancaster, PA), pp. 3-26.

Liang, C., Sun, F. P., and Rogers, C. A. (1993a). "An Impedance Method for Dynamic Analysis of Active Material Systems," Proceedings: 34th SDM Conference, La Jolla, CA, 19-21 April, pp. 3587-3599. Also in ASME J. Vibra. Acoust. 116(1), 120-128 (January 1994).

Liang, C., Sun, F. P., and Rogers, C. A. (1993b). "Coupled ElectricMechanical Analysis of Piezoelectric Ceramic Actuator Driven SystemsDetermination of the Actuator Power Consumption and System Energy Transfer," Proceedings, SPIE Conference on Smart Structures and Materials '93, 31 Jan.-4 Feb. Albuquerque, NM, pp. 286-298. Also in J. Intelligent Mater. Sys. Struct. 5, 12-20, 1994.

Liang, C., Sun, F. P. Rogers, C. A., and Gu, Y. (1994). "Determination of the Power Consumption of Stacked PZT Actuator-Driven Underwater Plates for Active Structural Acoustic Control," Proceedings: 35th SDM Conference and Adaptive Structures Forum, Hilton Head, SC, 21-22 April, pp. 73-82. 
Rogers, C. A. (1990). "Active Vibration and Structural Acoustic Control of Shape Memory Alloy Hybrid Composites: Experimental Results," J. Acoust. Soc. Am. 90, 2803-2811.

Rossi, A., Liang, C., and Rogers, C. A. (1993). "Impedance Analysis of Piezoceramic Actuator Driven Systems-An Application to Cylindrical Structure," Proceedings: 34th SDM Conference, La Jolla, CA, 19-21 April 1993, pp. 3618-3624.

Roussos, L. A. (1985). "Noise Transmission Loss of a Rectangular Plate in an Infinite Baffle," NASA Tech. Paper 2398.

Sandman, B. E. (1977). "Fluid-loaded Vibration of an Elastic Plate Carrying a Concentrated Mass," J. Acoust. Soc. Am. 61, 1503-1510.

Saunders, W. R., Robertshaw, H. H., and Rogers, C. A. (1990). "Experimental Studies of Structural Acoustic Control for a Shape Memory Alloy Composite Beam," AIAA-90-1090-CP, Proceedings of the 31st SDM Conference, Washington, DC.

Sonti, V. R., Jones, J. D., and Herrick, R. W. (1991). "Active Vibration
Control of Thin Cylindrical Shells Using Piezo-Electric Actuators," Proceedings: Recent Advances in Active Control of Sound and Vibration, 15-17 April (Technomic, Lancaster, PA), pp. 27-38.

Wang, B. T. (1991). "Active Control of Sound Transmission/Radiation from Elastic Plates Using Multiple Piezoelectric Actuators," Ph.D. dissertation, Dept. of Mech. Eng., Virginia Polytechnic Institute and State University (June 1991).

Zhou, S. W., Liang, C., and Rogers, C. A. (1994a). “An Electro-Mechanical Impedance Analysis to Predict Power Consumption of Piezoelectric Actuators Integrated with Thin Plates," Proceedings: 35th SDM Conference and Adaptive Structure Forum, April 21-22, Hilton Head, SC, pp. 259269.

Zhou, S. W., Liang, C., and Rogers, C. A. (1994b). "A Dynamic Design Method of Piezoelectric Actuators Integrated with Adaptive Structures," Proceedings: the Second International Conference on Intelligent Materials, Williamsburg, Virginia (June 1994). 